\title{
Expression of Human Leukocyte Antigen-G in Placental Tissues from Pregnant Women with Preeclampsia
}

\author{
Yongmei Li ${ }^{1}$, , Yubin Zhang ${ }^{2}$, Jinfang Wang ${ }^{1}$ \\ ${ }^{1}$ Department of Gynecology, Binzhou People's Hospital, Binzhou, P.R. China \\ ${ }^{2}$ Department of Nuclear Medicine, Binzhou People's Hospital, Binzhou, P.R. China
}

Email address:

liyongmei2020@163.com (Yongmei Li)

${ }^{*}$ Corresponding author

\section{To cite this article:}

Yongmei Li, Yubin Zhang, Jinfang Wang. Expression of Human Leukocyte Antigen-G in Placental Tissues from Pregnant Women with Preeclampsia. American Journal of Life Sciences. Vol. 8, No. 1, 2020, pp. 14-18. doi: 10.11648/j.ajls.20200801.13

Received: May 26, 2020; Accepted: June 22, 2020; Published: June 29, 2020

\begin{abstract}
Objective: To study the gene and protein expressions of human leukocyte antigen-G (HLA-G) in the placental tissues from healthy women in late pregnancy and pregnant women with preeclampsia (PE), and to explore the relationship between HLA-G and PE. Methods: Immunohistochemistry, Western blot and RT-PCR were used to detect the expressions of HLA-G protein and mRNA in the maternal placenta of 60 pregnant women with PE (including 30 cases of mild PE and 30 cases of severe PE) and 30 healthy full-term pregnant women (control group) respectively. Results: HLA-G had its expression mainly on the surface of trophoblast cells, and its response range and reactivity of its immunologic tissue were further reduced and weakened as the patient's condition was aggravated. The protein expression of placental HLA-G in each PE group was significantly lower than control group, with the difference statistically significant (all $\mathrm{P}<0.05$ ). Compared with mild $\mathrm{PE}$ group, the protein expression level of HLA-G was lower in severe PE group $(\mathrm{P}<0.05)$. The Spearman's correlation analysis for HLA-G and PE severity showed that the correlation coefficient was $-0.892(\mathrm{rs}=-0.892)(\mathrm{P}<0.05)$, indicating that the expression of HLA-G protein was significantly negatively correlated to the severity of PE. The mRNA expression level of placental HLA-G of each PE group was significantly lower than that of control group, and the difference was statistically significant (all $\mathrm{P}<0.05$ ). But the mRNA expression level of HLA-G was significantly lower in severe $\mathrm{PE}$ group than in mild $\mathrm{PE}$ group $(\mathrm{P}<0.05)$. Conclusion: The decreased expression of HLA-G in placenta may be involved in the pathogenesis of PE, and can also reflect the severity of this disease.
\end{abstract}

Keywords: HLA-G, Placenta, Preeclampsia, Pregnancy

\section{Introduction}

Preeclampsia $(\mathrm{PE})$ is a pregnancy-specific multi-system disease that threatens the health of both mothers and babies with unknown causes. It is a relatively severe type in the classification of pregnancy-induced hypertension (PIH), one of the common gestational diseases as well as one of the main causes for maternal mortality. Too shallow invasion of trophoblast cells to endometrium and the remodeling disorders of uterine spiral arteries are its main pathological features $[1,2]$. It may be involved in maternal, placental and fetal factors, including abnormal trophoblast invasion, immune dysfunction, endothelial cells injury, genetic and nutritional factors $[3,4]$. But there is no one single factor that can explain the pathogenesis of PE's incidence.

Studies have found that human leukocyte antigen-G (HLA-G) plays an important role in maintaining maternal-fetal immune tolerance and regulating trophoblast infiltration function, and its abnormal expression participates in the incidence of hypertensive disorders in pregnancy [5]. The original transcripts of HLA-G can produce a number of isomer mRNAs through alternative splicing to respectively encode different protein subtypes, of which HLA-G1, G2, G3 and G4 are membrane-bound proteins, while HLA-G5, G6 and G7 are soluble proteins [6].

This study aims to detect the expression, distribution and differences of HLA-G gene and protein between the placenta tissues from pregnant women with PE and healthy women in late pregnancy so as to explore the relationship between HLA-G and the pathogenesis of PE. 


\section{Methods}

\subsection{Clinical Data}

Thirty patients with mild PE, 30 patients with severe PE and 30 healthy pregnant women who were admitted and delivered in the Obstetrical Department of our hospital from Jan. 2012 to Mar. 2013 were selected, all of which were single pregnancies. They were divided into mild PE group, severe PE group and control group respectively. The ages of mild and severe PE groups and control group were 20-32 $(26.7 \pm 5.2)$ years old, $21-33(26.9 \pm 5.8)$ years old and $20-33(27.2 \pm 5.6)$ years old respectively. Gestational weeks were $(36.7 \pm 2.2),(37.2 \pm 1.8)$ and $(37.5 \pm 1.6)$ respectively. There were no statistically significant differences in the age, gestational week and times of gestation and delivery among the three groups by balancing test (all $\mathrm{P}>0.05$ ). The diagnostic criteria for $\mathrm{PE}$ were referred to the relevant literatures, previous hypertension, diabetes, heart disease, immune system disorders, liver and kidney diseases and the history of other chronic diseases excluded [7].

\subsection{Sample Treatment}

For all cases, 3 to 4 pieces of tissue, about $1 \mathrm{~cm}^{3}$ for each, in the central part of maternal surface of placenta attached to the root of umbilical cord were taken by sterile scissor after the delivery of placenta, and the regions of hemorrhage, necrosis and calcification were avoided. One piece was rinsed with sterile normal saline (containing $0.5 \%$ DEPC water) to remove the blood on it, and placed into freezing tube processed and sterilized by DEPC water. The other pieces were rapidly placed into liquid nitrogen after being rinsed with normal saline, and transferred to $-70^{\circ} \mathrm{C}$ refrigerator after overnight for standby use. Another piece of fresh placenta of about $1 \mathrm{~cm}^{3}$ was taken and fixed in $3 \%$ paraformaldehyde for immunohistochemical detection.

\subsection{Detection of HLA-G protein Expression in Placental Tissues by Immunohistochemistry Method (SP Method)}

The placental tissue pieces fixed in 3\% paraformaldehyde mentioned above were conducted conventional dehydration, paraffin-embedding and section at $5 \mu \mathrm{m}$. The slides were cleaned and coated with APES to prevent section flaking, 3 slices in each piece for immunohistochemical staining using the SP method. The main steps were as follows: The slices were incubated with mouse anti-HLA-G monoclonal antibody (1: 200 , U.S. Sigma) overnight at room temperature and then biotinylated rabbit anti-mouse $\operatorname{IgG}(1: 100$, Amresco) at room temperature for $6 \mathrm{~h}$. DAB/ $\mathrm{H}_{2} \mathrm{O}_{2}$ was used for color development and hematoxylin for mild counterstaining. The primary antibody was replaced with antibody diluent as the negative control, and the reaction was negative. Five high-power fields $(\times 200)$ were randomly selected under optical microscope, observed and photographed. The absorbance values of HLA-G immune reaction products on each slice were measured using Image-Pro Plus Version 6.0 color image analysis system and made semi-quantitative statistical analysis.

\subsection{Detection of HLA-G Protein Expression in Placental Tissues by Western Blot}

Placental tissue $(500 \mathrm{mg}$ ) was added $5 \mathrm{ml}$ of cell lysis buffer, homogenized, and centrifuged at $3000 \mathrm{r} / \mathrm{min}$ and $4^{\circ} \mathrm{C}$ for $10 \mathrm{~min}$ to take the supernatant which was separated by electrophoresis for protein samples. The proteins were transferred to NC membrane by constant current at $275 \mathrm{~mA}$ for $1 \mathrm{~h}$ for Western blot. The samples were incubated with mouse anti-HLA-G monoclonal antibody (1: 2000, U.S. Sigma) at $4^{\circ} \mathrm{C}$ overnight, with mouse anti- $\beta$-actin monoclonal antibody (1: 1000 , USA sigma) as internal reference, and horse radish peroxidase (HRP)-labeled rabbit anti-mouse IgG (1: 1000, Shanghai RICKY Bio-tech Co., Ltd.) at room temperature for $6 \mathrm{~h}$. NC membrane was made color development with $\mathrm{DAB} / \mathrm{H}_{2} \mathrm{O}_{2}$ and the results were obtained by scanner. The bio-electrophoresis image analysis system (OLYMPUS, Japan) was used to analyze the absorbance values of bands. The target protein level was expressed by the ratio of the average absorbance values of the target band and the internal reference $\beta$-actin, and then made analysis.

\subsection{Detection of HLA-G mRNA Expression in Placental Tissues by RT-PCR}

Placental tissue $(500 \mathrm{mg}$ ) was extracted total RNA by Trizol method (Trizol kit was purchased from Invitrogen, USA). HLA-G mRNA level was detected by RT-PCR. Primers were designed and synthesized by Shanghai Jianglai Bio-tech Co., Ltd. $\beta$-Actin was amplified as an internal reference. The annealing temperatures for the sequences of all target gene primers, base positions, lengths of amplified fragments and PCR amplification were shown in Table 1.

Table 1. PCR amplification primer sequences and fragments.

\begin{tabular}{lll}
\hline & Primer sequence & Length \\
\hline HLA-G & Upstream primer 5'-ACAGGCACACAATACCCGGC-3' & 497 bp \\
& Downstream primer 5'-CTCCTAGTGGCCTTGATCTTC-3' & 30 \\
$\beta$-Actin & Upstream primer 5'-ATGAGTCATCTGTCACTCGG-3' & \\
& Downstream primer 5'-TGAGTCATTTGTCACCCGGA-3' & 341 bp \\
\hline
\end{tabular}

PCR amplification products were conducted $3.0 \%$ agarose gel electrophoresis, and gel imaging system (Labnet) was used for analysis of absorbance values of bands. The $\beta$-actin mRNA level was expressed by the ratio of the mean absorbance values of the target gene and $\beta$-actin, and then made semi-quantitative analysis.

\subsection{Statistical Analysis}

The measurement data were expressed as $\mathrm{X} \pm \mathrm{S}$ and analyzed by SPSS 15.0. Means of groups were compared by F test. Two groups were compared by LSD method. Correlation was subjected to Spearman's rank correlation analysis. $\mathrm{P}<0.05$ was 
considered statistically significant.

\section{Results}

\subsection{Immunoreactive Expressions of HLA-G in Placental Tissues}

HLA-G protein had expression in the placental tissues of
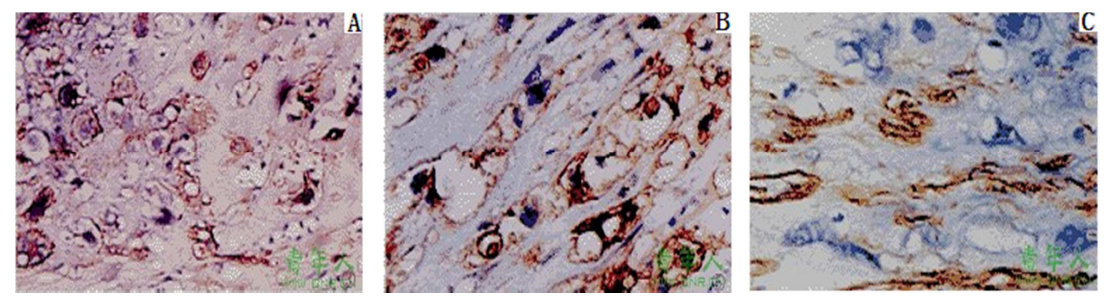

Figure 1. Immunoreactive expressions of HLA-G in placental tissues (×400). A: Control group; B: mild PE group; C: severe PE group.
The results of semi-quantitative analysis showed that compared with control group, the positive staining of placental HLA-G immunoreaction in PE groups became shallow, and the average absorbance values were reduced, therefore, the protein expression level of placental HLA-G in each PE group was decreased, with the difference statistically significant (all $\mathrm{P}<0.05$ ). Compared with mild $\mathrm{PE}$ group, the positive staining of placental HLA-G immunoreaction in severe PE group was more shallow, so the protein expression level of placental HLA-G was lower in severe PE group than in mild PE group, with the difference statistically significant $(\mathrm{P}<0.05)$ (Table 2).

Table 2. Absorbances of HLA-G immunoreaction products.

\begin{tabular}{lll}
\hline Group & n & HLA-G expression \\
\hline Control & 30 & $0.85 \pm 0.12$ \\
Mild PE & 30 & $0.57 \pm 0.09^{*}$ \\
Severe PE & 30 & $0.26 \pm 0.08^{* \#}$ \\
\hline
\end{tabular}

Compared with the control group, $* \mathrm{P}<0.05$; compared with mild $\mathrm{PE}$ group, $\# \mathrm{P}<0.05$.

\subsection{HLA-G protein Expression in Placental Tissues Detected by Western Blot}

The molecular weights of HLA-G and $\beta$-actin proteins were $97 \mathrm{kD}$ and $56 \mathrm{kD}$ respectively. The absorbance values of bands in each group were corrected with $\beta$-actin as internal reference and then made analysis. The results showed that the protein expression level of placental HLA-G in each PE group was significantly lower than control group, with the difference statistically significant (Figure 2), with the difference statistically significant (all $\mathrm{P}<0.05$ ). Compared with mild $\mathrm{PE}$ group, the protein expression level of HLA-G was lower in severe $\mathrm{PE}$ group $(\mathrm{P}<0.05)$ (Figure 2). The Spearman's correlation analysis for HLA-G and PE severity showed that the correlation coefficient was $-0.892(\mathrm{rs}=-0.892)(\mathrm{P}<0.05)$, indicating that the expression of HLA-G protein was significantly negatively correlated to the severity of PE. In other words, HLA-G protein expression level can be further decreased as the patient's condition was aggravated. both control group and PE groups. The positive products of HLA-G immunoreaction were brown granular or punctate, expressed mainly on the surface of trophoblast cells. Its response range and reactivity of its immunologic tissue were further reduced and weakened as the patient's condition was aggravated (Figure 1).

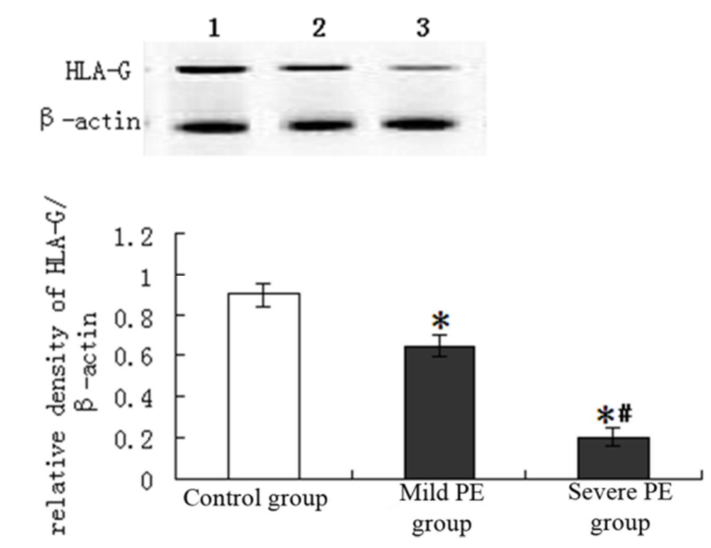

Figure 2. HLA-G protein expression in placental tissues detected by Western blot. 1: Control group; 2: mild PE group; 3: severe PE group. Compared with the control group, $* P<0.05$; compared with mild PE group, $\# P<0.05$.

\subsection{HLA-G mRNA in Placental Tissues Detected by RT-PCR}
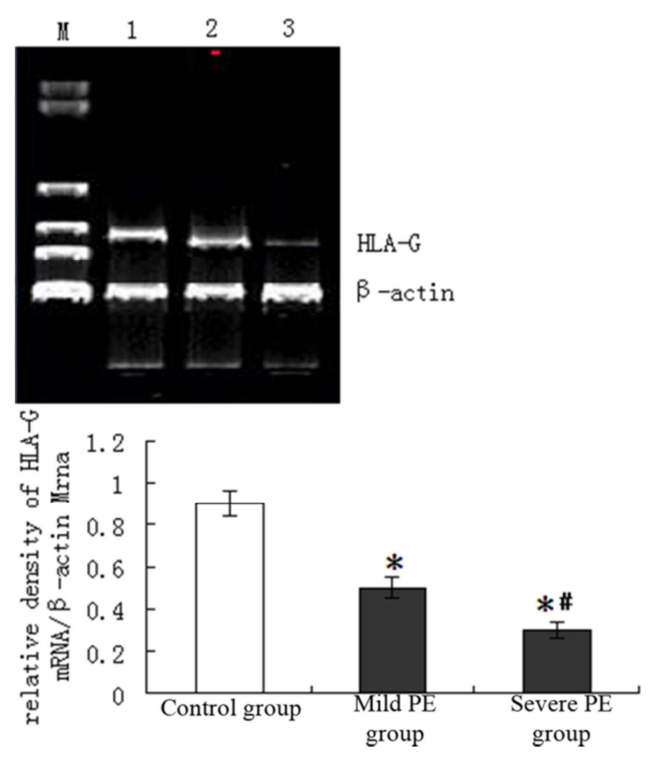

Figure 3. HLA-G mRNA in placental tissues detected by RT-PCR. M: Marker; 1: control group; 2: mild PE group; 3: severe PE group. Compared with the control group, ${ }^{*} P<0.05$; compared with mild $P E$ group, $\# P<0.05$. 
The lengths of amplified fragments of HLA-G and $\beta$-actin mRNAs were $497 \mathrm{bp}$ and $341 \mathrm{bp}$ respectively, with clear bands. The absorbance values of bands in each group were corrected with $\beta$-actin as internal reference and then made analysis. The results showed that the mRNA expression level of placental HLA-G in each PE group was significantly lower than that of control group, and the difference was statistically significant (all $\mathrm{P}<0.05$ ). But the mRNA expression level of HLA-G was significantly lower in severe PE group than in mild $\mathrm{PE}$ group $(\mathrm{P}<0.05)$ (Figure 3 ).

\section{Discussion}

HLA-G belongs to a non-classical HLA- I type (HLA- I b) gene family, which is located on human chromosome 6 short arm 6p21.3, with encoding membrane binding heavy chain (HC). The whole $\mathrm{HC}$ consists of the extracellular region (containing $\alpha 1, \alpha 2$ and $\alpha 3$ domains), transmembrane region and cytoplasmic region. $\mathrm{HC}$ is bound with $\beta 2$ microglobulin into a functional protein by non-covalent bond. HLA-G transcript is composed of eight exons, seven introns and two untranslated regions, of which exon 1 encodes the signal peptide, exons 2-4 extracellular $\alpha 1-\alpha 3$ domains and exons 6-8 cytoplasmic region [8]. Its original transcript can generate seven kinds of isomer mRNA through alternative splicing, which can encode seven kinds of protein molecule: the four membrane-bound molecules are HLA-G1 (containing 3 extracellular functional domains), HLA-G2 (lacking $\alpha 2$ domain), HLA-G3 (lacking $\alpha 2$ and $\alpha 3$ domains), HLA-G4 (lacking $\alpha 3$ domains) respectively, and the three soluble molecules are HLA-G5, HLA-G6 and HLA-G7 respectively. Both the intron 4 contained in HLA-G5 and HLA-G6 transcripts and the intron 2 contained in HLA-G7 mature mRNA have terminating codons, resulting in the transmembrane and cytoplasmic regions unable to be translated, so as to turn into a soluble form [9]. HLA-G has the features of low-degree polymorphism and limitation of distribution, mainly expressed in extravillous trophoblast [10]. Studies have found that HLA-G molecule can inhibit the activity of maternal NK cells and protect trophoblast cells from their killing, and it can also induce the generation of Th2-type cytokines and promote Th1/Th2-type equilibrium to migrate towards the Th2-type direction conducive to pregnancy, taking trophic effects on trophoblast cells [11]. In recent years, it has been found that its non-full-length molecules, i.e. HLA-G2, HLA-G3 and HLA-G4 can also inhibit the cytolytic effects of NK cells and antigen-specific CTL. In individuals with homozygous deletion of HLA-G1, these subtypes play a leading role in inducing maternal-fetal immune tolerance [12]. Therefore, most scholars believe that HLA-G is an immune-tolerance molecular, which can maintain normal pregnancy through the induction of immune tolerance of maternal-fetal interface, while its abnormal expression may lead to pathological pregnancy [13].

Some studies have found that compared with normal placental tissue, the protein expression of placental HLA-G was significantly decreased in patients with severe PE [14].
Thus, it can be speculated that due to the defect of HLA-G protein expression, trophoblast cells have a reduced ability of inhibiting the attack of maternal decidual NK cells and some cytotoxic cytokines, so that extravillous trophoblast cannot be effectively implanted into the maternal spiral arteries, which can result in vascular casting blockage and placental hypoperfusion, causing a series of adverse consequences, and ultimately developing to hypertensive disorders in pregnancy [15].

In recent years, more and more studies have shown that trophoblast infiltration is the key for the incidence and progress of PE. The implantation of fertilized egg of normal pregnancy into uterine cavity requires twice physiological intrusions of trophoblast cells to destroy the muscle elastic fibers, smooth muscle fibers and nerve tissues on vessel wall, so that the spiral artery appears progressive dilation, and loses its elasticity and its reactivity to vasoconstrictor substances, and then it succumbs to physiological expansion. This is spiral arterial recasting, which can be found in the decidual layer and part of the superficial muscle segments [16, 17]. But extravillous trophoblast cells have superficial infiltration in $\mathrm{PE}$, and uterine spiral arteries, muscular spiral arteries in particular, are imperfectly rebuilt in the decidua of uteroplacental bed and superficial muscle segment. The lumen diameters of most spiral arteries are only half of those in healthy pregnant women in the same period, and in addition, the arterial muscular layer is still thick, vascular endothelial cells have not yet been replaced with trophoblast cells, so there are rare endovascular trophoblast cells in the spiral arteries in the muscular layer [18, 19]. Zhao et al. studied the placental bed biopsies from normal pregnant women and those with PE, and found that HLA-G had sustained high expression in placental cells in the former, but on the contrary, HLA-G had low expression in the latter [20]. Thus, it can be inferred that the primary reason for the incidence of PE lies in that placental cells in PE may experience abnormal differentiation at the molecular level, with reduced trophoblast infiltration, resulting in poor spiral arterial recasting and shallow placental implantation.

In this study, immunohistochemistry, Western blot and RT-PCR were used to study the differences in the expression of placental HLA-G in healthy pregnant women and those with $\mathrm{PE}$ from transcription to translation in both qualitative and quantitative way. The experimental results show that HLA-G protein had expression in the placental tissues of both control group and PE groups, mainly on the surface of trophoblast cells. However, the expression levels of HLA-G protein in PE placentas were significantly lower than that of normal group, and as the condition of PE was aggravated, their levels were further decreased markedly. The RT-PCR results show that the expression levels of HLA-G mRNA transcription were consistent with those of HLA-G protein in all the three groups. Therefore, it can be inferred that HLA-G has decreased impression in placental tissues from pregnant women with PE. On the one hand, it may reduce the binding of HLA-G with the extracellular matrix ligand fibronectin, affect the normal transition of trophoblast cells from adhesion 
phenotype to invasion phenotype, and inhibit the ability of trophoblast invasion, while on the other hand, HLA-G decline may affect the activation of its downstream signaling molecules, so as to cause the abnormality of multiple signal transduction pathways of trophoblast cells, affect their growth, proliferation, differentiation, apoptosis and invasion, etc., eventually leading to decreased trophoblast invasion, which is closely related to the pathogenesis of PE.

\section{Conclusion}

In summary, low HLA-G expression plays a major role in the incidence of hypertensive disorders in pregnancy. Its expression level monitoring is conducive to the early diagnosis of this disease, and regulating its gene expression may also become a new target for the treatment of hypertensive disorders of pregnancy, so as to provide a new idea for its pathogenesis, diagnosis and treatment.

\section{References}

[1] Laskowska M, Laskowska K, Oleszczuk J. Differences in the Association between Maternal Serum Homocysteine and ADMA Levels in Women with Pregnancies Complicated by Preeclampsia and/or Intrauterine Growth Restriction. Hypertens Pregnancy 2020; 31: 83-93.

[2] Scholten RR, Hopman MT, Sweep FC, et al. Co-occurrence of cardiovascular and prothrombotic risk factors in women with a history of preeclampsia. Obstet Gynecol 2019; 121: 97-105.

[3] Scazzocchio E, Figueras F, Crispi F, et al. Performance of a first-trimester screening of preeclampsia in a routine care low-risk setting. Am J Obstet Gynecol 2019; 208: 203-210.

[4] Warner CM, Lampton PW, Newmark JA, Cohen J. Symposium: innovative techniques in human embryo viability assessment. Soluble human leukocyte antigen-G and pregnancy success. Reprod Biomed Online 2018; 17: 470-485.

[5] Rizzo R, Andersen AS, Lassen MR, et al. Soluble human leukocyte antigen-G isoforms in maternal plasma in early and late pregnancy. Am J Reprod Immunol 2019; 62: 320-338.

[6] Bezerra Maia E Holanda Moura S, Marques Lopes L, Murthi P, da Silva Costa F. Prevention of preeclampsia. J Pregnancy 2017; 15: $90-96$

[7] Walpole NG, Kjer-Nielsen L, Kostenko L, et al. The structure and stability of the monomorphic HLA-G are influenced by the nature of the bound peptide. J Mol Biol 2018; 397: 467-480.

[8] Castelli EC, Mendes-Junior CT, Deghaide NH, et al. The genetic structure of 3'untranslated region of the HLA-G gene: polymorphisms and haplotypes. Genes Immun 2019; 11: 134-141.
[9] Kolte AM, Steffensen R, Nielsen HS, Hviid TV, Christiansen OB. Study of the structure and impact of human leukocyte antigen (HLA)-G-A, HLA-G-B, and HLA-G-DRB1 haplotypes in families with recurrent miscarriage. Hum Immunol 2019; 71 (5): 482-488.

[10] Agaugué S, Carosella ED, Rouas-Freiss N. Role of HLA-G in tumor escape through expansion of myeloid-derived suppressor cells and cytokinic balance in favor of Th2 versus Th1/Th17. Blood 2019; 117: 7021-7031.

[11] Chen BG, Xu DP, Lin A, Yan WH. NK cytolysis is dependent on the proportion of HLA-G expression. Hum Immunol 2018; 74: 286-289.

[12] Darmochwal-Kolarz D, Kolarz B, Rolinski J, Leszczynska-Gorzelak B, Oleszczuk J. The concentrations of soluble HLA-G protein are elevated during mid-gestation and decreased in pre-eclampsia. Folia Histochem Cytobiol 2017; 50: 286-291.

[13] Zhang Z, Li Y, Zhang LL, Jia LT, Yang XQ. Association of 14 bp insertion/deletion polymorphism of the HLA-G gene in father with severe preeclampsia in Chinese. Tissue Antigens 2017; 80: 158-164.

[14] Tan CY, Chong YS, Loganath A, et al. Possible gene-gene interaction of KIR2DL4 with its cognate ligand HLA-G in modulating risk for preeclampsia. Reprod Sci 2019; 16: 1135-1143.

[15] Moreau P, Contu L, Alba F, et al. HLA-G gene polymorphism in human placentas: possible association of $\mathrm{G}^{*} 0106$ allele with preeclampsia and miscarriage. Biol Reprod 2018; 79: 459-467.

[16] Zhou X, Zhang GY, Wang J, Lu SL, Cao J, Sun LZ. A novel bridge between oxidative stress and immunity: the interaction between hydrogen peroxide and human leukocyte antigen $\mathrm{G}$ in placental trophoblasts during preeclampsia. Am J Obstet Gynecol 2018; 206: 7-16.

[17] Suzuki S. The benefit of labor induction for preeclampsia beyond 37 weeks' gestation. J Matern Fetal Neonatal Med 2018; 23: $1072-1074$.

[18] Faupel-Badger JM, Fichorova RN, Allred EN, et al. Cluster analysis of placental inflammatory proteins can distinguish preeclampsia from preterm labor and premature membrane rupture in singleton deliveries less than 28 weeks of gestation. Am J Reprod Immunol 2019; 66: 488-494.

[19] Zhao SC, Li ZB, He TQ, Yu CZ. Relationship between the expression of human leukocyte antigen $\mathrm{G}$ and preeclampsia. Zhonghua Fu Chan Ke Za Zhi 2019; 46: 758-762.

[20] Liu CM, Chang SD, Cheng PJ. Relationship between prenatal care and maternal complications in women with preeclampsia: implications for continuity and discontinuity of prenatal care. Taiwan J Obstet Gynecol 2019; 1: 576-582. 